\title{
Case Report \\ Early Stage of Chronic Kidney Disease with Renal Injury Caused by Hypertension in a Dog
}

\author{
Akira Yabuki, Keijiro Mizukami, Mariko Sawa, Sawane Mitani, and Osamu Yamato \\ Laboratory of Veterinary Clinical Pathology, Department of Veterinary Medicine, Kagoshima University, \\ 1-21-24 Korimoto, Kagoshima 890-0065, Japan \\ Correspondence should be addressed to Akira Yabuki, yabu@agri.kagoshima-u.ac.jp \\ Received 6 July 2011; Accepted 3 August 2011 \\ Academic Editor: C. Hyun \\ Copyright ( 92011 Akira Yabuki et al. This is an open access article distributed under the Creative Commons Attribution License, \\ which permits unrestricted use, distribution, and reproduction in any medium, provided the original work is properly cited. \\ A 10-year-old spayed female Papillon weighing $4.0 \mathrm{~kg}$ presented with a history of persistent hematuria and pollakiuria. Concurrent \\ bladder calculi, a mammary gland tumor, and nonazotemic early stage of chronic kidney disease with contracted kidneys were \\ noted in this dog. The dog underwent cystectomy, unilateral mastectomy, and intraoperative renal biopsy. On the basis of \\ histopathological analysis of renal biopsy results, it was suspected that renal injury of the dog was caused by persistent hypertension, \\ and a follow-up examination revealed severe hypertension. The dog was treated with a combination of an angiotensin-converting \\ enzyme inhibitor and calcium channel blocker. The treatment produced a good outcome in the dog, and there has been no \\ progression of the chronic kidney disease for over 2 years.
}

\section{Introduction}

The close association between progression of kidney damage and systemic hypertension is well known in human and small animal medicine [1-5]. However, in cases when kidney disease with systemic hypertension is diagnosed from clinical symptoms and laboratory test results, a renal biopsy is required to determine whether renal injury is induced by persistent hypertension or whether it is the preexisting kidney disease. This report presents a case of a dog with nonazotemic early stage of chronic kidney disease probably caused by hypertensive renal injury.

\section{Case Presentation}

A 10-year-old spayed female Papillon weighing $4.0 \mathrm{~kg}$ presented with a 3-week history of persistent hematuria and pollakiuria. Bladder calculi were observed on radiographic and ultrasonographic examination. A firm, small mass in the left mammary gland was also detected on palpation. Marked leukocytosis $\left(90.0 \times 10^{3} / \mu \mathrm{L}\right)$ and mild erythrocytosis $(9.07 \times$ $\left.10^{6} / \mu \mathrm{L}\right)$ with increased hematocrit $(62 \%)$ were shown in complete blood count. Although mild increase of blood urea nitrogen $(10 \mathrm{mmol} / \mathrm{l})$ was shown in blood chemical analysis, the plasma creatinine concentration (pCre) was found to be within the reference range at $71 \mu \mathrm{mol} / 1$. Urinalysis could not be performed because of an empty bladder. Small-sized kidneys, one 2.2 (right kidney) and the other 1.8 (left kidney) times the length of the second lumbar, were observed during radiographic examination, and reduced corticomedullary differentiation with hyperechogenicity in both kidneys was also noted during the ultrasonographic examination. These findings from diagnostic imaging suspected that the dog has nonazotemic chronic kidney disease (CKD), namely, stage 1 CKD in the International Renal Interest Society (IRIS) classification. The leukocyte concentration decreased to $10.2 \times$ $10^{3} / \mu \mathrm{L}$ after 1 week of antibiotic therapy. The dog owner gave informed consent for the surgical interventions and the renal biopsy for definitive diagnosis of the kidney disease, and the dog underwent cystectomy, unilateral mastectomy, and intraoperative renal biopsy. Follow-up examinations demonstrated that the bladder calculi were struvites, and the mammary mass was a benign mixed tumor. The following description focuses on the diagnosis and management of the kidney disease.

Renal Biopsy. A portion of the renal tissue was excised from the left kidney using a $14 \mathrm{G}$ semi-automatic biopsy needle (Stericut, TSK, Tochigi, Japan). The tissue sample was 


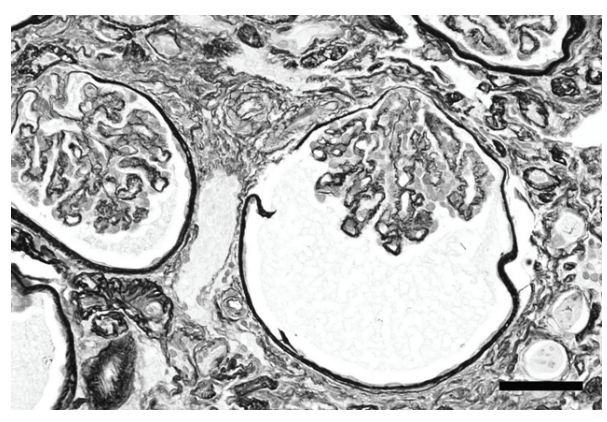

(a)

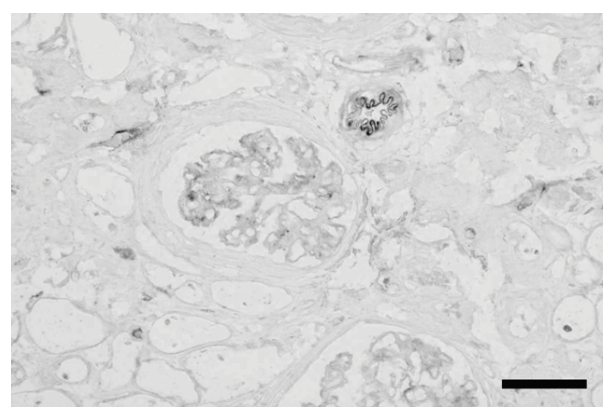

(c)

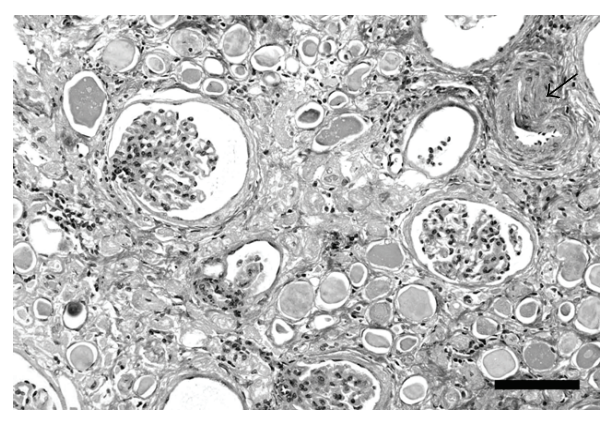

(b)

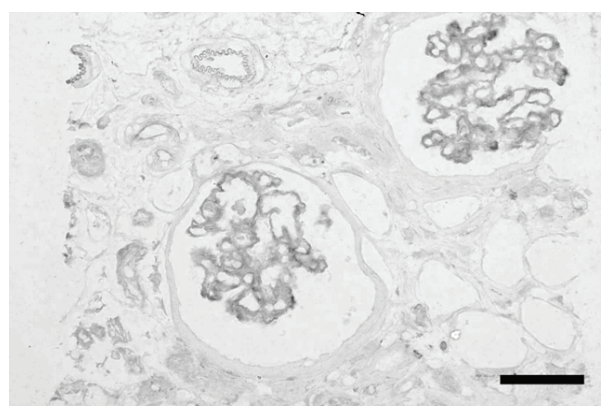

(d)

FIGURE 1: Light micrographs in renal biopsy analysis. (a) Periodic acid methenamine-silver stain; collapse of glomerular tufts, wrinkling of glomerular basement membrane (GBM), and thickening of Bowman's capsule are observed. (b) Masson's trichrome stain; severe interstitial fibrosis is observed. Most of the tubular epithelia are flattened, and their lumens are filled with casts. Hyaline degeneration, luminal narrowing, and thickening of the tunica media are seen in a small artery (arrow). (c) IgM detection; granular positive-signals are observed in the capillary wall and mesangial area in the glomeruli. Internal elastic membrane of the small artery shows a positive reaction. (d) Complement C3 detection; linear positive signals are observed in the glomeruli. Bars; 50 (a, c, and d) and 100 (b) $\mu \mathrm{m}$.

fixed with $10 \%$ neutral buffered formalin and embedded in paraffin with a routine procedure, and $2 \mu \mathrm{m}$ thick sections were cut serially. For observation using a conventional light microscope, sections were stained with hematoxylineosin, periodic acid Schiff, periodic acid methenaminesilver, Masson's trichrome, and elastica van Gieson stains. Immunohistochemistry was performed using goat polyclonal antibodies against dog immunoglobulin (Ig) G, IgA, IgM, and complement $\mathrm{C} 3$ as primary antibodies (Bethyl Laboratories, Montgomery, Tex, USA).

Histopathology. There were about 20 glomeruli in the observed sections. Diffusely and globally observed changes in the glomeruli were wrinkling of the glomerular basement membrane, collapse of the glomeruli, and thickening of Bowman's capsule (Figure 1). Severe interstitial fibrosis and tubular atrophy with periodic acid Schiff-positive cast formation were observed (Figure 1). Mononuclear cell infiltration by lymphocytes and plasma cells was also observed in the interstitium. Hyaline degeneration, thickening of tunica media, and luminal narrowing were observed in the small arteries and arterioles (Figure 1). Positive signals for IgM and complement C3 were observed in the glomeruli, small arteries, and arterioles (Figure 1). No positive signals were detected for $\operatorname{IgG}$ or $\operatorname{Ig} \mathrm{A}$.
These histopathological findings resembled those of hypertensive renal injury in human [6], and this findings suspected that systemic hypertension has persisted in the dog. Although systolic blood pressure (SBP) could not be measured previously because this dog has an aggressive behavior, evaluation of the SBP was retried according to the Doppler method (Model 811-B; Parks Medical Electronics, LasVegas, Nev, USA). Urinary protein/creatinine ratio (UPC) was determined after confirmation of non-bacteriuria. From the careful evaluation according to the standardized procedure $[7,8]$, the dog's SBP was considered over $180 \mathrm{~mm}$ $\mathrm{Hg}$. The UPC was found to be 0.34 . Therefore, the dog was clinically diagnosed as IRIS stage 1 CKD (pCre: $<125 \mu \mathrm{mol} / \mathrm{l}$ ) with severe hypertension (SBP: $>180 \mathrm{~mm} \mathrm{Hg}$ ) and borderline proteinuria (UPC: $0.2-0.4$ ). Since renal injury induced by persistent hypertension was suggested from the findings of renal biopsy, antihypertensive therapy was initiated.

Changes in SBP and pCre are shown in Figure 2. The SBP did not fall below $180 \mathrm{~mm} \mathrm{Hg}$ after administration of the angiotensin-converting enzyme inhibitor (ACEI) benazepril $(0.69 \mathrm{mg} / \mathrm{kg}$ p.o. once or twice daily) treatment. Although the calcium channel blocker (CCB) amlodipine $(0.35 \mathrm{mg} / \mathrm{kg}$ p.o. once daily) was added to the benazepril treatment, this combination of antihypertensive agents only induced temporarily antihypertensive action. Informed consent was 


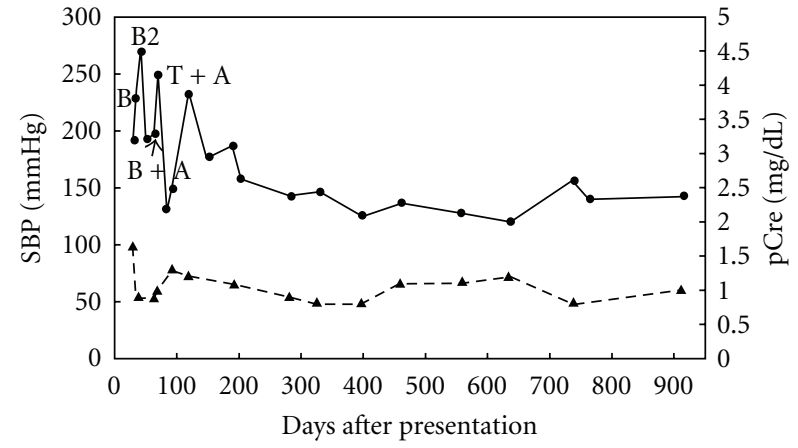

FIGURE 2: Changes in the systolic blood pressure (SBP; solid line) and plasma creatinine concentration (pCre; dashed line). B: benazepril $(0.69 \mathrm{mg} / \mathrm{kg}$, once daily); B2: benazepril $(0.69 \mathrm{mg} / \mathrm{kg}$, twice daily); A: amlodipine $(0.35 \mathrm{mg} / \mathrm{kg}$, once daily); T: temocapril $(0.11 \mathrm{mg} / \mathrm{kg}$, once daily).

obtained from the dog owner for replacing the type of ACEI, that is, for replacing benazepril with temocapril $(0.11 \mathrm{mg} / \mathrm{kg}$ p.o. once daily), before increasing the dosage of amlodipine or adding of a different class of antihypertensive agents. This change in ACEI was effective; the dog's SBP gradually decreased to within the normotensive range and became stable. The dog has been treated with a combination of amlodipine and temocapril for over 2 years and has managed to stay clinically healthy having IRIS stage $1 \mathrm{CKD}$ with nonproteinuria (UPC: $<0.2$ ).

\section{Discussion}

In human medicine, the term hypertensive nephrosclerosis (HN) was used to describe the renal injury caused by essential hypertension [9]. Although human $\mathrm{HN}$ is usually diagnosed solely on clinical grounds, importance of the pathological analysis of renal biopsy results has been pointed out recently $[10,11]$. The most typical features of $\mathrm{HN}$ are vascular lesions such as hyaline degeneration and sclerosis. The most common glomerular change of $\mathrm{HN}$ is an ischemic change featured by collapse of the capillary tufts with wrinkling of the basement membrane [6]. Tubulointerstitial lesions are frequently observed in the cases of $\operatorname{HN}[6,12]$. Thickening of Bowman's capsule with collapse of glomerular tufts and interstitial fibrosis are regarded as changes in the case of long-standing hypertension. Although histopathological evidence from the renal biopsy was sufficient to diagnose the renal injury of the dog to be caused by persistent hypertension, hypertension in the present case could not be diagnosed as essential. Although laboratory test results and clinical symptoms did not suggest the presence of predisposing causes of canine secondary hypertension such as diabetes, hyperadrenocorticism, acute renal failure, or hypothyroidism, the dog was not diagnosed as the essential hypertension in the strict sense, because concurrent urolithiasis and mammary gland tumor were demonstrated in the dog.

In the present case, immunopositive signals for IgM and C3 were clearly detected in the glomeruli, small arteries, and arterioles. Although immunohistochemical examinations have not been fully performed even in human $\mathrm{HN}$, depositions of IgM and C3 have been considered as the most common findings; these depositions are not considered to be the primary cause of glomerular damage but as nonspecific accumulation and deposition in areas of injured tissue [6].

ACEI and CCB are commonly used as antihypertensive agents in dogs and cats [7]. In the present case, coadministration of ACEI and CCB improved systemic hypertension without increasing pCre or exacerbating the dog's condition. Interestingly, a tangible and gradual decrease in the blood pressure was seen after the type of ACEI used was changed from benazepril to temocapril. Temocapril belongs to the newest generation of ACEIs, which are commercially available for dog therapy in our country; temocapril results in long-lasting preferential biliary excretion and has high bioavailability. However, it is difficult to explain why temocapril was more effective than benazepril in this dog. Similar to temocapril, benazepril is also an ACEI that causes long-lasting and preferential biliary excretion. Significant antihypertensive effects of benazepril in dogs with CKD involving systemic hypertension have already been demonstrated experimentally and clinically in previous reports $[9$, 13]. Although the findings from the present case indicated that temocapril is an effective antihypertensive agent, its superiority to other ACEIs has not yet been demonstrated.

\section{References}

[1] M. Adamczak, M. Zeier, R. Dikow, and E. Ritz, "Kidney and hypertension," Kidney International, Supplement, vol. 61, no. 80, pp. S62-S67, 2002.

[2] G. L. Bakris, M. Williams, L. Dworkin et al., "Preserving renal function in adults with hypertension and diabetes: a consensus approach," American Journal of Kidney Diseases, vol. 36, no. 3, pp. 646-661, 2000.

[3] F. Jacob, D. J. Polzin, C. A. Osborne et al., "Association between initial systolic blood pressure and risk of developing a uremic crisis or of dying in dogs with chronic renal failure," Journal of the American Veterinary Medical Association, vol. 222, no. 3, pp. 322-329, 2003.

[4] R. E. Jepson, J. Elliott, D. Brodbelt, and H. M. Syme, "Effect of control of systolic blood pressure on survival in cats with systemic hypertension," Journal of Veterinary Internal Medicine, vol. 21, no. 3, pp. 402-409, 2007.

[5] M. Tozawa, K. Iseki, C. Iseki, K. Kinjo, Y. Ikemiya, and S. Takishita, "Blood pressure predicts risk of developing endstage renal disease in men and women," Hypertension, vol. 41, no. 6, pp. 1341-1345, 2003.

[6] J. L. Olson, "Renal disease caused by hypertension," in Pathology of The Kidney, JC Jennette, JL Olson, MM Schwartz, and FG Silva, Eds., pp. 937-990, Lippincott Williams \& Wilkins, Philadelphia, Pa, USA, 6th edition, 2007.

[7] S. Brown, C. Atkins, R. Bagley et al., "Guidelines for the identification, evaluation, and management of systemic hypertension in dogs and cats," Journal of Veterinary Internal Medicine, vol. 21, no. 3, pp. 542-558, 2007.

[8] R. A. Henik, M. K. Dolson, and L. J. Wenholz, "How to obtain a blood pressure measurement," Clinical Techniques in Small Animal Practice, vol. 20, no. 3, pp. 144-150, 2005.

[9] M. Mishina and T. Watanabe, "Development of hypertension and effects of benazepril hydrochloride in a canine remnant 
kidney model of chronic renal failure," Journal of Veterinary Medical Science, vol. 70, no. 5, pp. 455-460, 2008.

[10] E. R. S. P. Caetano, R. Zatz, L. B. Saldanha, and J. N. Praxedes, "Hypertensive nephrosclerosis as a relevant cause of chronic renal failure," Hypertension, vol. 38, no. 2, pp. 171-176, 2001.

[11] G. S. Hill, "Hypertensive nephrosclerosis," Current Opinion in Nephrology and Hypertension, vol. 17, no. 3, pp. 266-270, 2008.

[12] H. Ono and Y. Ono, "Nephrosclerosis and hypertension," Medical Clinics of North America, vol. 81, no. 6, pp. 1273-1288, 1997.

[13] J. Tenhündfeld, P. Wefstaedt, and I. J. A. Nolte, “A randomized controlled clinical trial of the use of benazepril and heparin for the treatment of chronic kidney disease in dogs," Journal of the American Veterinary Medical Association, vol. 234, no. 8, pp. 1031-1037, 2009. 

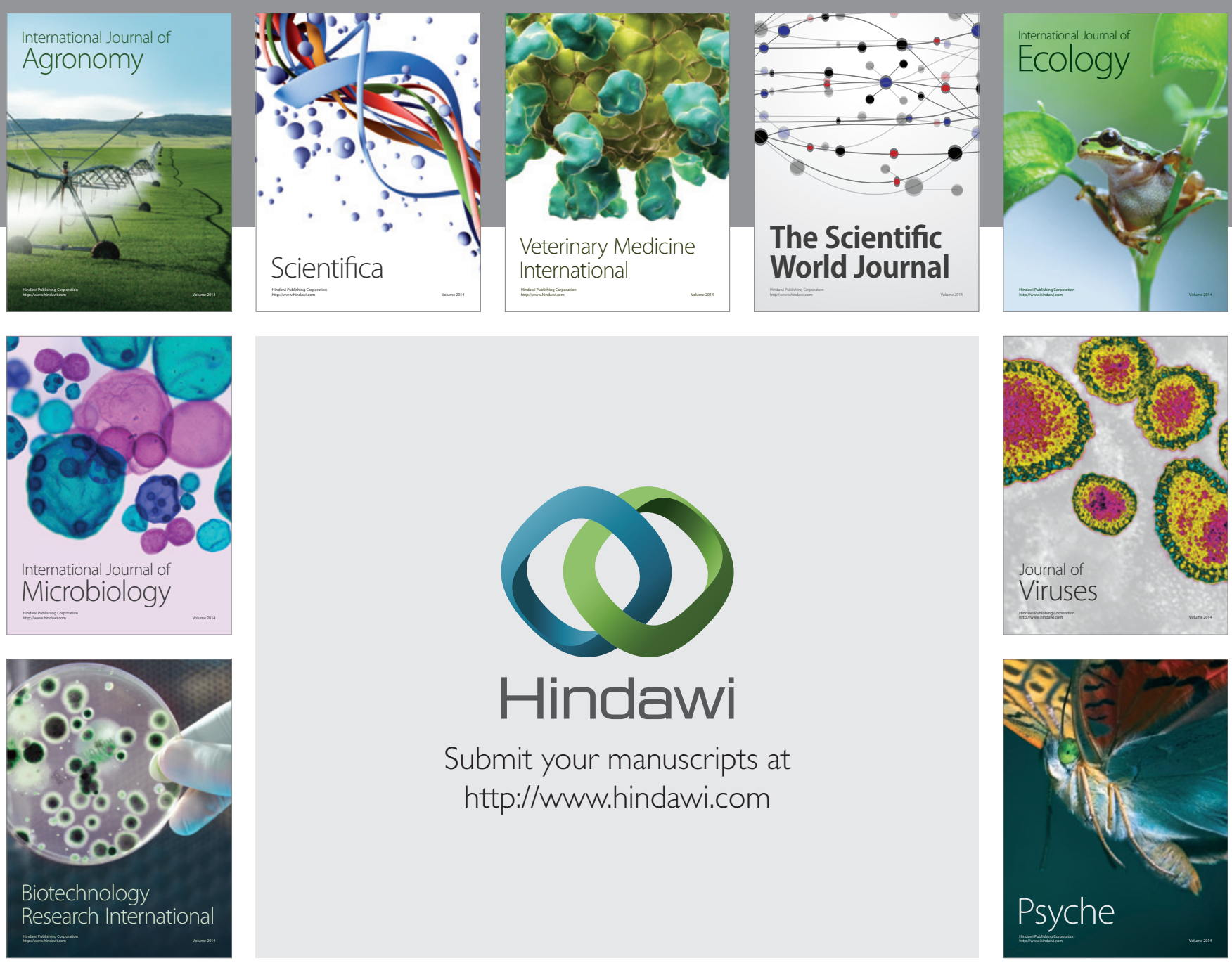

Submit your manuscripts at

http://www.hindawi.com
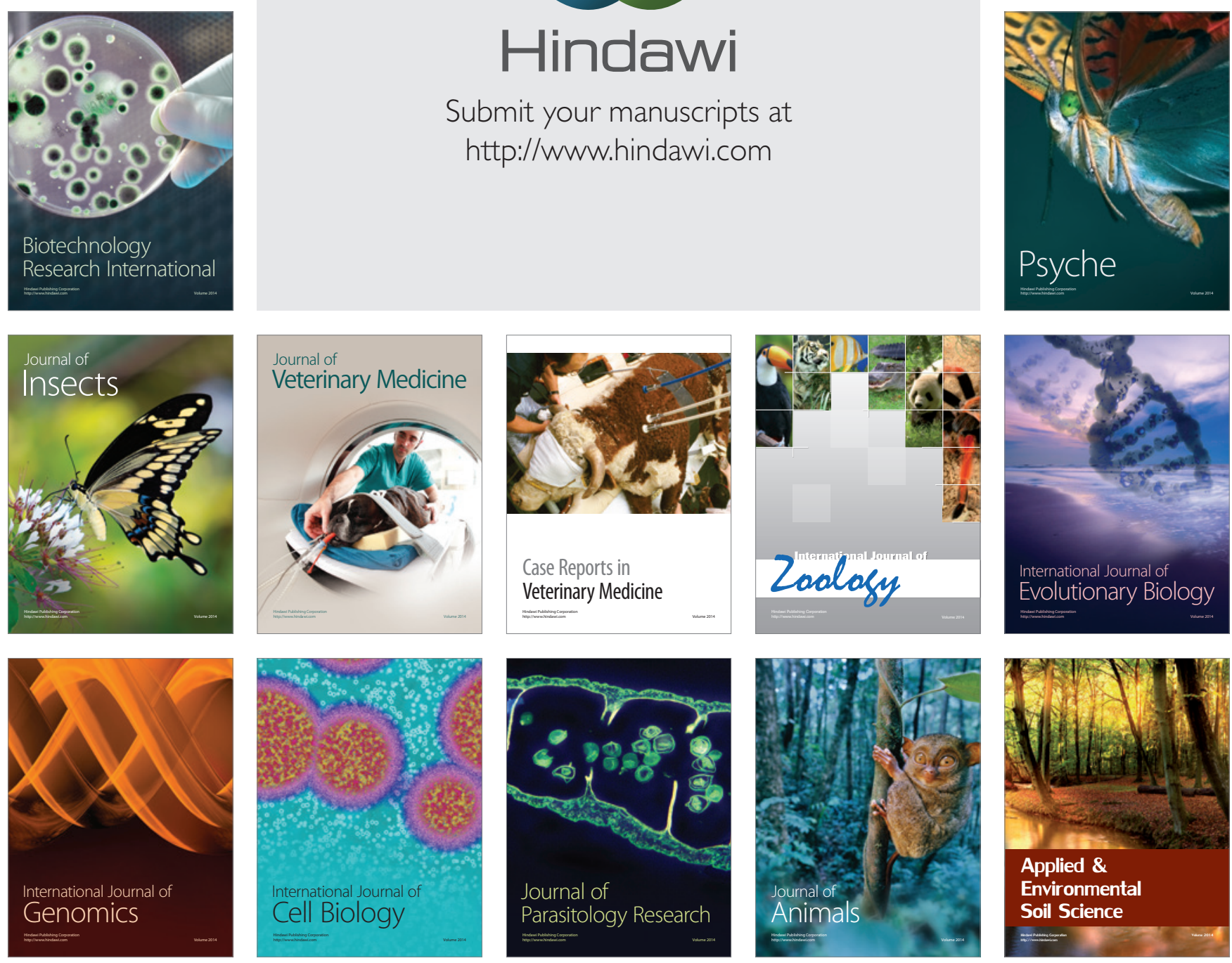